\title{
artigo
}

\section{Influência da equoterapia na qualidade de vida de idosos caracterizados com a classificação internacional de funcionalidade - CIF}

Influence of therapeutic horseback riding in life quality of elderlies classified by the international classification of functioning, disability, and health - ICF

Influencia de la equoterapia en la calidad de vida de los ancianos caracterizados con la clasificación internacional de funcionalidad - CIF

\section{RESUMO}

Objetivo: Caracterizar idosos ativos através de Core Set da Classificação Internacional de Funcionalidade (CIF) para saúde de idosos e avaliar a qualidade de vida por meio do The 36-Item Short Form Health Survey- SF-36 na terapia assistida com equinos. Métodos: Trata-se de um estudo observacional analítico transversal, onde 10 idosos ativos entre 60 à 80 anos realizaram 16 sessões de terapia assistida com equinos, com duração de 30 minutos cada. Para avaliação foi utilizado o questionário da CIF, o SF-36 e o Mini Exame do Estado Mental - MEEM. Foi realizado análise estatística descritiva e também foi utilizado o teste t pareado. Resultados: $\mathrm{O}$ MEEM pré intervenção não demonstrou declínio cognitivo. Na CIF, os domínios I, II e III ficaram com qualificadores predominantemente em 0 e 2 , já o domínio IV as pontuações ficaram com qualificadores entre 3 e 4. Conclusão: A terapia assistida com equinos em idosos é uma alternativa terapêutica efetiva na melhora da qualidade de vida.

DESCRITORES: Classificação Internacional de Funcionalidade., Equoterapia Assistida., Qualidade de vida., Idosos.

\section{ABSTRACT}

Objective: The objective of this study was to classify active elderlies' health and life quality through the Core set of the International Classification of functioning, disability and health (ICF) and the 36-Item Short Form Health Survey (SF-36), in therapeutic horseback riding. Methods: An observational, analytical, transversal study was carried out with ten elderlies of 60 to 80 years old. All individuals performed 16 therapeutic horseback riding session of 30 minutes each. ICF, SF-36 and the Mini-mental State Examination (MMSE) were utilized to evaluate the elderlies. Data were analyzed by descriptive statistical analysis and paired Student's t-test. Results: MMSE pre-intervention demonstrated no cognitive decline. In ICF, domains I, II and II were predominantly coded with qualifiers 0 and 2, and domain IV with qualifiers 3 and 4. Conclusion: Therapeutic horseback riding for elderlies is an effective therapeutic alternative for the maintenance and improvement of life quality.

DESCRIPTORS: International Classification of Functionality., Assisted Riding Therapy., Quality of life., Elderly.

\section{RESUMEN}

Objetivo: caracterizar a las personas mayores activas a través del Conjunto básico de la Clasificación Internacional de Funcionalidad (ICF) para la salud de las personas mayores y evaluar la calidad de vida a través de la Encuesta de salud de forma breve de 36 ítems - SF-36 en terapia asistida equina. Métodos: Este es un estudio analítico transversal observacional, en el que 10 personas mayores activas entre 60 y 80 años realizaron 16 sesiones de terapia asistida con caballos, con una duración de 30 minutos cada una. El cuestionario ICF, el SF-36 y el Mini examen del estado mental - MEEM se utilizaron para la evaluación. Se realizó un análisis estadístico descriptivo y también se utilizó la prueba t pareada. Resultados: el MMSE previo a la intervención no demostró deterioro cognitivo. En el ICF, los dominios I, II y III tenían calificadores predominantemente en 0 y 2, mientras que en el dominio IV los puntajes tenían calificadores entre 3 y 4 . Conclusión: la terapia asistida por caballos en los ancianos es una alternativa terapéutica efectiva para mantener y / o mejorar la calidad de vida.

DESCRIPTORES: Clasificación Internacional de Funcionalidad., Equitación Asistida., Calidad de vida., Anciano.

RECEBIDO EM: 12/12/2020 APROVADO EM: 07/01/2021 


\section{Angela Dubiela Julik}

Docente do curso de Fisioterapia, Universidade Estadual do Centro-Oeste, PR, Brasil. ORCID: 0000-0001-7375-6771

\section{Andressa Leticia Miri}

Mestranda em Ciências da Reabilitação, Universidade Estadual de Londrina, PR, Brasil. ORCID: 0000-0002-9057-5339

\section{Ana Carolina Dorigoni Bini}

Docente do curso de Fisioterapia, Universidade Estadual do Centro-Oeste, PR, Brasil. ORCID: 0000-0003-1717-9249

\section{Patricia Pacheco Tyski Suckow}

Docente do curso de Fisioterapia, Universidade Estadual do Centro-Oeste, PR, Brasil.. ORCID: 0000-0002-5022-7612

\section{Eliane Gonçalves de Jesus Fonseca}

Docente do curso de Fisioterapia, Universidade Estadual do Centro-Oeste, PR, Brasil.. ORCID: 0000-0002-6540-6111

\section{Felipe Figueiredo Moreira}

Discente em Fisioterapia, Universidade Estadual do Centro-Oeste, PR, Brasil.. ORCID: 0000-0002-6625-4084

\section{Emerson Carraro}

Docente do curso de Farmácia, Universidade Estadual do Centro-Oeste, PR, Brasil. ORCID: 0000-0001-5420-2300

\section{INTRODUÇÃO}

0 envelhecimento caracteriza-se pela deterioração progressiva na função das células, tecidos e órgãos, levando ao declínio das funções fisiológicas, como perda de massa muscular, redução do fluxo sanguíneo, comprometimento no sistema imune, diminuição da capacidade energética, alteração da função cognitiva e capacidade reduzida para responder a estímulos de estresse. Estes declínios relacionados à idade culminam no início de doenças, entre elas, sarcopenia, doenças cardiovasculares, câncer, obesidade, diabetes e doenças neurodegenerativas ${ }^{1}$.

As questões referentes à velhice e ao processo de envelhecimento têm despertado amplamente o interesse da sociedade de um modo geral, em função do rápido envelhecimento populacional que vem ocorrendo em diversos países, inclusive no Brasil. É possível dizer que isto se deve, basicamente, à diminuição da taxa de natalidade e ao aumento da expectativa de vida, proporcionada pelo progresso tecnológico em vários campos científicos ${ }^{2}$. Seja no âmbito de pesquisas ou de cuidados, o crescimento populacional direciona a atenção para propostas que visem à manutenção da saúde nessa populaçãa ${ }^{3}$.

$\mathrm{Na}$ abordagem do desenvolvimento comunitário e consequentemente em saúde pública, a diminuição da resiliência biológica e da plasticidade comportamental compatíveis com a senescência e com a fragilidade, acarretam aumento de perdas em saúde, funcionalidade física, funcionamento intelectual, atividade, motivação, participação social e bem-estar subjetivo, as quais podem ser agravadas por eventos estressantes gerados pela vulnerabilidade social, entre eles as necessidades não atendidas, e pelo duplo prejuízo de ser idoso e pertencer ao segmento social dos mais pobres e menos educados 4 .

Atualmente é consenso entre profissionais da saúde que a atividade física é um fator determinante no sucesso do processo do envelhecimento, cientificamente a relação entre qualidade de vida, atividade física e envelhecimento vem sendo analisado fre- quentemente, tal a sua relevância ${ }^{5}$.

A fisioterapia tem a importante missão da reabilitação e da promoção de saúde dos idosos, auxiliando tanto no alívio dos sintomas, quanto na promoção da independência funicional e na qualidade de vida ${ }^{6}$. Uma forma terapêutica é a equoterapia, a qual utiliza o cavalo como instrumento para promover educação e equitação em pessoas portadoras ou não de deficiência, trazendo para o praticante benefícios de âmbitos social, físico, psicológico e educacional ${ }^{7}$.

Devido a este método ser aplicado em ambientes abertos, acaba tornando a terapia mais agradável e prazerosa, capaz de superar danos comportamentais, motores, sensoriais e cognitivos, todos melhorados por causa das oscilações causadas na garupa do cavalo, exigindo que haja participação de todas as partes do corpo do indivíduo para realização dos exercícios ${ }^{8,9}$.

A fim de propor uma alternativa de promoção de saúde aos idosos, buscando contribuir para a qualidade de vida, longevidade e inclusão social, o objetivo do presente estudo foi caracterizar idosos ativos e avaliar a quali- 


\section{artigo}

Julik, A.D.; Miri, A.L.; Bini, A.C.D.; Suckow, P.P.T.; Fonseca, E.G.J.; Moreira, F.F.; Carraro, E.;

Influência da equoterapia na qualidade de vida de idosos caracterizados com a classificação internacional de funcionalidade - CIF

dade de vida comparando pré e pós intervenção da terapia assistida com equinos.

\section{MÉTODOS}

O presente trabalho foi realizado em 2018 e trata-se de um estudo observacional analítico transversal. Para sua viabilização, foram seguidas as normas preconizadas pelo Conselho Nacional de Saúde (CNS) para pesquisas junto a seres humanos e da Comissão Nacional de Ética em Pesquisa (CONEP), estabelecidas na Resolução n. 466 de 12 de dezembro de 2012 da Comissão Nacional de Ética em Pesquisa (CONEP); e pelo Conselho Federal de Fisioterapia e Terapia Ocupacional (COFFITO) através da Resolução n. 10 de 03 julho de 1978.

Este estudo foi aprovado pelo Comitê de Ética em Pesquisa da Universidade Estadual do Centro Oeste - UNICENTRO, pelo parecer número 1814780.

Os atendimentos ocorreram em lugar adequado, com animais treinados e com equipamentos adequados para garantir a segurança das participantes durante os atendimentos.

A população alvo constituiu-se de idosos com idade entre 60 e 80 anos, matriculados na UNATI, vinculada a Universidade Estadual do Centro-Oeste (UNICENTRO), do município de Guarapuava, no estado do Paraná.

A amostra foi obtida por conveniência através do convite a idosos disponíveis e interessados em participar da pesquisa, por contato telefônico, resultando em amostra final de 10 participantes.

Como critérios de inclusão foram considerados idosos matriculados na UNATI, com idade entre 60 e 90 anos, praticantes de atividades físicas, idosos com capacidade para entender ordens simples, capacidade de realizar atividades de vida diária de forma independente, montar à cavalo de forma independente e apresentar atestado médico liberando para a prática da equoterapia.

Quanto aos critérios de exclusão, indivíduos que utilizem cadeira de rodas e as contra indicações absolutas para a equoterapia segundo a Associação Nacional de equoterapia- ANDE BRASIL (2004): obesos, instabilidade atlanto-axial, osteoporose moderada à severa, dor durante a terapia, luxação de quadril, distrofia muscular, epilepsia, amputação pélvica, hipertensão arterial não controlada, quadros inflamatórios/ infecciosos, tumores, alergias, idosos institucionalizados, com comprometimentos neurológicos, pessoas com mais de $90 \mathrm{Kg}$ (peso do cavaleiro maior que $20 \%$ do peso do animal compromete a qualidade da biomecânica do passo do cavalo) e que apresentaram fobia ao animal.

O estudo foi realizado no Serviço de Hipoterapia e Prática Paraequestre da Universidade Estadual do Centro Oeste -UNICENTRO e da Clínica Escola de Fisioterapia, localizada no Campus CEDETEG, com o grupo de idosos participantes da Universidade Aberta da Terceira Idade (UNATI).

Inicialmente, os participantes receberam o Termo de Consentimento Livre e Esclarecido expondo todos os riscos e benefícios da pesquisa, bem como os esclarecimentos importantes para o praticante, documento de liberação de dados e imagem e Termo de Ciência e Compromisso (ANEXO I). Somente após aprovação do participante e sua assinatura em todos os documentos, iniciou-se a coleta de dados.

Para realização dos testes os idosos foram convidados a se dirigirem ao Serviço de Hipoterapia e Prática Paraequestre da UNICENTRO, sendo iniciada as intervenções 7 dias após a avaliação. Ao final da $16^{a}$ sessão de equoterapia, os idosos foram instruídos a manterem suas atividades diárias sem alterações e a permanecerem sem atividade física orientada durante o período do estudo e retornarem após 7 dias e realizarem os Pós-testes.

\section{A coleta de dados deu-se com instru- mentos de avaliação distintos:}

Para caracterização da amostra foi utilizado a ficha de avaliação (ANEXO II) onde continham questões sobre, idade, estado civil, escolaridade, profissão, renda familiar, peso, altura e IMC. Foram colhidas informações sobre patologias preexistentes e medicamentos em que o idoso fazia uso, além de atividades físicas que realizavam na UNATI, a frequência e, se havia possibili- dade de interromper momentaneamente esses exercícios.

Com a finalidade de caracterizar a amostra quanto à funcionalidade, foi aplicado pré intervenção um Cor Set da CIF específico para a saúde física de idosos, em 4 domínios e 28 categorias/subcategorias.

Para atestar amostra sem declínio cognitivo, optou-se por aplicar pré e pós intervenção o Mini Exame do Estado MentalMEEM. Também, foi aplicado pré e pós intervenção o questionário para avaliar a qualidade de vida com o The 36-Item Short Form Health Survey- SF-36.

Para a aplicação da CIF um grupo de seis acadêmicos do quinto ano de Fisioterapia da UNICENTRO receberam um treinamento, após conclusão deste deu-se a coleta de dados.

A aplicação ocorreu na maior parte do tempo na forma de uma entrevista, onde o avaliador lia a categoria e o indivíduo avaliado respondia classificando a resposta em qualificadores: sem comprometimento algum (0), ligeiro comprometimento (1), moderado comprometimento (2), grave comprometimento (3) ou totalmente comprometido (4); as respostas variavam de acordo com a pergunta, mas seguia sempre a mesma linha de raciocínio.

Apenas duas categorias eram diferentes: uma, no domínio funções do corpo - com a finalidade de avaliar a função proprioceptiva - onde havia 4 testes de propriocepção, os quais o idoso deveria completar corretamente todos para ser classificado com qualificador máximo naquela categoria; outra, ainda no domínio funções do corpo, para classificar o tônus de todos os músculos do corpo, a tarefa era palpar a musculatura do idoso e classificá-la como comprometida ou não e qual o grau de comprometimento ${ }^{10}$.

O MEEM criado por Folstein et al. (1975), foi utilizado para avaliar a função cognitiva dos idosos. Suas questóes abordam a orientação temporal e espacial, registro e recordação de três palavras, atenção, cálculo, linguagem e capacidade construtiva visual.

O MEEM é composto por diversas questôes agrupadas em sete categorias, sendo que cada uma delas objetiva avaliar 
déficits das funções cognitivas específicas: orientação para o tempo (5 pontos), orientação para o local (5 pontos), registro de três palavras ( 3 pontos), atenção e cálculo ( 5 pontos), lembrança de três palavras (3 pontos), linguagem (8 pontos) e capacidade construtiva visual (1 ponto). O escore pode variar de um mínimo de 0 até o máximo de 30 pontos.

O declínio cognitivo é classificado de acordo com a escolaridade: analfabetos o ponto de corte é de 19; 1 à 3 anos de escolaridade ponto de corte de 23; 4 à 7 anos ponto de corte de 24 e mais de 7 anos de estudo ponto de corte de 28 pontos.

Para avaliação da qualidade de vida foi utilizado o questionário genérico The 36Item Short Form Health Survey (SF-36), sendo um dos questionários de qualidade de vida mais utilizados cientificamente ${ }^{11}$. Os participantes respondiam ao questionário que contém 36 itens, dos quais 35 encontram-se agrupados em oito dimensóes (capacidade funcional, dor, aspectos físicos, aspectos emocionais, aspectos sociais, saúde mental, vitalidade e estado geral de saúde) e um último item que avalia a mudança de saúde no tempo. Para cada dimensão, os itens do SF-36 são codificados, agrupados e transformados em uma escala de zero (pior estado de saúde) a 100 (melhor estado de saúde) ${ }^{12}$.

Foram realizadas 16 sessões de equoterapia, com duração de 30 minutos cada, 2 vezes semanais. Para os atendimentos foram utilizadas duas éguas, Maragata, crioula, de cor zaina de 20 anos que transpista ao passo, e Querida de cor preta, mestiça que ao passo também transpista. Esses animais são devidamente treinados para prática equoterápica e assistidos atentamente pela equipe de medicina veterinária da universidade, com as devidas autorizações sanitárias.

Os materiais utilizados para montaria foram: capacetes, selas, mantas, cabeçadas, cabresto, estribo, cilhão e freio. Para a terapia: tambores, bolas, bastões e cones, além de materiais de escritório para registro das sessões.

Os atendimentos contavam com diferenciação do trajeto, que contou com uma boa arborização e também com agradável oportunidade de margear o lago. $\mathrm{O}$ ambiente proporcionou durante a prática equoterápica o contato com a natureza.

O local de espera para o praticante também oferece grande espaço sob arvoredo de mata nativa ao ar livre. A rampa de acesso à montaria é adaptada ao local facilitando aos praticantes em espera visualizar os outros praticantes em montaria.

A sessão foi dividida em: exercícios de aquecimento, com duração de 5 minutos; exercícios de treinamento específico, com duração de 20 minutos; e 5 minutos finais de relaxamento.

A seleção das atividades a serem realizadas nas sessões baseou-se nas sugestões de atividades propostas nos cursos promovidos pela ANDE-BRASIL (2004), bem como na experiência da prática clínica do mediador.

Foi realizado no aquecimento: exercícios respiratórios; alongamentos; exercícios de consciência corporal; elevação de braços alternados, simultâneos; rotação do tronco; encontro das mãos à frente, acima e atrás.

$\mathrm{Na}$ segunda etapa, realizou-se os seguintes exercícios:

a) Mudanças de direção ritmadas: com dissociação de cinturas pélvica e escapular;

b) Variações de piso (terra, cascalho fino, asfalto e gramado): esta variação de piso tem como objetivo ativação dos barorreceptores, estimulando assim, a propriocepção;

c) Variações de terreno (plano, acidentado e inclinado): a variação de terreno tem como objetivo intensificar movimentos de anteroversão e retroversão pélvica;

d) Volteio: são exercícios de passagens posturais realizados sob o cavalo, que se movimenta em círculos. $\mathrm{Na}$ realização de volteio os idosos alternavam as posições de montaria: sentando lateralmente na manta; posteriormente sentando na postura invertida; sentava lateralmente novamente, porém na lateral contrária a utilizada anteriormente, finalizando com a montaria clássica. Intensificando assim, a ação do sistema vestibular, da coordenação motora e fortalecimento muscular;

e) Subir e descer nos estribos: esse exercício tem como objetivo principal promover o fortalecimento muscular de membros superiores e inferiores;

f) Grito de incentivo: ao final de cada exercício o mediador falava "Uipe" e os idosos respondiam "Rá", com intuito de descontrair a terapia e realizar uma expiração forçada, aumentando assim o volume corrente de ar nos pulmões.

$\mathrm{Na}$ fase final da prática realizou-se o relaxamento ou volta à calma, que constitui de exercícios de descontração psíquica e muscular, com objetivo de favorecer a diminuição da contração muscular excessiva, além de educar as sensações proprioceptivas. Também realizaram-se exercícios respiratórios, alongamento e interação afetiva com o cavalo ainda montado.

A análise dados foi realizada no SPSS, versão 25.0. Os dados foram apresentados em média e desvio padrão. Inicialmente aplicou-se o teste de Shapiro-Wilk para confirmação da hipótese de normalidade. As variáveis que violaram a normalidade foram ajustadas matematicamente (BoxCox). As medidas pré e pós foram comparadas pelo teste t pareado, sendo adotado como nível de significância $\mathrm{p}<0,05$.

\section{RESULTADOS}

Na tabela 1 encontra-se as características $\mathrm{da}$ amostra $(\mathrm{N}=10)$ : idade, gênero, peso, altura, IMC, escolaridade, renda e estado civil. Os valores estão dispostos em porcentagem, sendo a amostra predominantemente do sexo feminino, casados (as), idade média de 70, 6 anos, com renda mensal estável (4 mil reais) e IMC acima do normal $(26,63)$.

Para a análise dos dados da CIF foi utilizada a estatística descritiva a fim de obter a frequência relativa (\%), a partir da frequência absoluta com que os qualificadores apareciam na amostra.

Os resultados da classificação da amostra foram divididos em duas tabelas, as quais apresentam dois domínios cada. A pontu- 


\section{artigo}

Julik, A.D.; Miri, A.L.; Bini, A.C.D.; Suckow, P.P.T.; Fonseca, E.G.J.; Moreira, F.F.; Carraro, E.;

Influência da equoterapia na qualidade de vida de idosos caracterizados com a classificação internacional de funcionalidade - CIF

ação dos qualificadores está representada em porcentagem para cada subcategoria e todos os domínios da CIF.

Na tabela 2 estão expostos o Domínio I da CIF, que representa as funções do corpo. Suas subcategorias correspondem: nível de energia; qualidade do sono; função vestibular do movimento; função proprioceptiva; sensação de dor; resistência física geral; capacidade aeróbica; mobilidade generalizada das articulações; força, tônus e resistência de todos os músculos do corpo. Observa-se que os qualificadores estão majoritariamente entre 0 e 2 , ou seja, os indivíduos possuem nenhum problema a moderado problema.

Também estão representados na tabela 2 o Domínio II da CIF que equivale as estruturas do corpo, está subdividida em: coração, ossos, articulações e músculos. Nota-se que os valores estão predominantemente em 0 e 1 , os quais indicam nenhum ou ligeiro problema estrutural do corpo.

A tabela 3 demonstra em porcentagem o Domínio III da CIF, o qual indica atividade e participação. Suas subcategorias envolvem: concentração e atenção; agachar-se; levantar-se; andar; utilização de transporte; garantir o próprio conforto físico; controle de dieta e forma física; relação com os pares e praticar esportes. Verifica-se que os idosos prevalentemente não possuem problemas em atividade e participação, ademais, nenhum relatou dificuldade em levantar-se e andar, favorecendo a pratica da equoterapia.

O Domínio IV da CIF referente aos fatores ambientais (tabela 5) foi subdividido em: medicamentos; produtos e tecnologias gerais para atividades; culturais, recreativas e esportivas e serviços de saúde. Adversamente aos Domínios I, II e III, o Domínio IV apresentou porcentagens dispostas em todos os qualificadores (predomínio $3 \mathrm{e}$ 4), desde nenhum problema vinculado aos fatores ambientais até completo problema.

Observa-se na tabela 4 os valores em média e desvio padrão do MEEM na pré e pós intervenção. A amostra apresenta cognição normal, e também não houve mudança relevante ao comparar pré e pós avaliação.

$\mathrm{Na}$ tabela 5 está presente em média e desvio padrão os valores da pontuação do

\section{Tabela 1- Características da amostra}

\section{Características}

$\begin{array}{lccc} & \text { Média } & \text { Mediana } & \text { DP } \\ \text { Idade } & 70,6 & 73 & 6,77 \\ \text { Gênero } & & & \\ \quad \text { Feminino (\%) } & 90 & & \\ \quad \text { Masculino (\%) } & 10 & & 11,6 \\ \text { Peso } & 69,93 & 65,15 & 0,13 \\ \text { Altura } & 1,69 & 1,57 & 1,6 \\ \text { Renda (mil reais) } & 3,9 & 4,54 \\ \text { IMC (Kg/cm }{ }^{2} \text { ) } & 20,63 & & \\ \text { Estado civil } & & & \\ \quad \text { Solteiro (\%) } & 10 & & \\ \quad \text { Casado (\%) } & 50 & & \\ \quad \text { Separado (\%) } & 20 & & \\ \quad \text { Viúvo (\%) } & 20 & & \\ \text { Fonte: apesquisadora DP: desvio pafrão } & & \end{array}$

\section{Tabela 2- Caracterização dos idosos pele CIF: Estrutura e Funçã do Corpo}

$$
\text { DOMÍNIO I - FUNÇÃO DO CORPO }
$$

Categorias/Subcategorias

B1300

B1343

B2352

B260

B280

B4550

B4551

B7102

B7306

B7356

B7402

57700

$\mathrm{S} 7701$

57702

Nivel de energia

Qualidade de sono

Função proprioceptiva

Sensação de dor

Resistência física geral

Capacidade aeróbica

Categorias/Subcategorias
Função vestibular do movimento

Mobilidade generalizada das articulações

Força de todos os músculos do corpo

Tônus de todos os músculos do corpo

Resistência de todos os músculos do corpo

\section{DOMÍNIO II - ESTRUTURA DO CORPO}

$\begin{array}{lcccccc}\text { S4100 } & \text { Coração } & 70 & 30 & 0 & 0 & 0 \\ \text { S7700 } & \text { Ossos } & 30 & 70 & 0 & 0 & 0 \\ \text { S7701 } & \text { Articulação } & 40 & 60 & 0 & 0 & 0 \\ \text { S7702 } & \text { Músculos } & 60 & 40 & 0 & 0 & 0\end{array}$

Fonte: a pesquisa 0- Nenhum problema apresentado; 1- Ligeiro problema apresentado; 2- Moderado problema apresentado; 3- Grave problema apresentado; 4 - Completo problema apresentado. $\mathrm{N}=10$ 
SF-36, nos domínios: capacidade funcional; aspectos físicos; dor; estado de saúde geral; vitalidade; aspectos sociais; aspectos emocionais e saúde mental. Obteve-se diferença estatística entre pré e pós intervenção na dor $(p=0,037)$ e nos aspectos sociais $(\mathrm{p}=0,008)$. Assim, ao obter redução significativa da dor e melhora nos aspectos sociais constata-se a efetividade da terapia com equinos em idosos.

\section{DISCUSSÃO}

Campos et al. (2014) realizaram um estudo transversal com 107 idosos pertencentes a unidades básicas de Minas Gerais (Brasil) buscando descrever o perfil sociodemográfico, bem como avaliar a relação de interdependência entre a qualidade de vida de idosos e atividade física (ginástica). Aplicaram alguns instrumentos, questionários e escala: Índice de Katz, Índice de Lawton-Brody, Mini exame do Estado Mental, Apgar Familiar; Questionário Internacional de Atividade Física, World Health Organization Quality of Life instrument-Old e a Escala de Depressão Geriátrica. Constataram que o perfil predominante eram mulheres, aposentadas, sem companheiro e com renda igual ou inferior a 622,00 reais. Ainda, verificaram que a qualidade de vida idosos é boa e influenciada pela prática de atividades físicas regulares, ausência de depressão, melhor capacidade cognitiva e funcionalidade familiar.

Araújo e Faro (2014) caracterizaram o perfil de 386 idosos participantes do Centro de Convivência do Idoso em São Paulo (Brasil) e investigaram as condições de saúde e desempenho nas atividades de vida diária conforme a Classificação Internacional de Funcionalidade, Incapacidade e Saúde e o Mini Exame do Estado Mental. Através dos dados notaram na amostra predomínio de mulheres, casadas, etnia auto declarante branca, com 5-10 anos de escolaridade, aposentadas, renda mensal de um salário mínimo, casa própria, meio de locomoção e atividade física preferida é a caminhada, com função cognitiva preservada. Constataram que a maioria das categorias da CIF era pouco comprometi-
Tabela 3- Caracterização dos idosos pele CIF: Atividade, Participação e Fatores Ambientais

DOMÍNIO III - ATIVIDADE E PARTICIPAÇÃO

\begin{tabular}{lcccccc} 
& & \multicolumn{5}{c}{ Qualificadores (\%) } \\
& Categorias/Subcategorias & 0 & $\mathbf{1}$ & $\mathbf{2}$ & $\mathbf{3}$ & $\mathbf{4}$ \\
D169 & Concentrar e atenção & 70 & 0 & 10 & 10 & 10 \\
D4101 & Agachar-se & 80 & 20 & 0 & 0 & 0 \\
D4104 & Levantar-se & 100 & 0 & 0 & 0 & 0 \\
D450 & Andar & 100 & 0 & 0 & 0 & 0 \\
D470 & Utilização de transporte & 60 & 30 & 10 & 0 & 0 \\
D5700 & Garantir o próprio conforto físico & 90 & 10 & 0 & 0 & 0 \\
D5701 & Controle e dieta e forma fícica & 90 & 0 & 10 & 0 & 0 \\
D7504 & Relação com os pares & 60 & 20 & 20 & 0 & 0 \\
D9201 & Praticar esportes & 60 & 30 & 10 & 0 & 0
\end{tabular}

DOMÍNIO IV- FATORES AMBIENTAIS

\section{Categorias/Subcategorias}

$\begin{array}{ccccccc}\text { E1101 } & \text { Medicamentos } & 0 & 70 & 30 & 0 & 0 \\ \text { E1400 } & \begin{array}{c}\text { Produtos e tecnologias gerais para atividades } \\ \text { Culturais recreativas e esportivas }\end{array} & 0 & 0 & 0 & 30 & 70 \\ \text { E5800 } & \text { Serviçoes de Saúde } & 0 & 10 & 10 & 30 & 50\end{array}$

Fonte: a pesquisa 0- Nenhum problema apresentado; 1- Ligeiro problema apresentado; 2- Moderado problema apresentado; 3- Grave problema apresentado; 4- Completo problema apresentado.

\section{Tabela 4- Mini Exame do Estado Mental - MEEM}

$\begin{array}{ccc}\text { MEEM } & \text { Média } & \text { Desvio padrão } \\ \text { Pré-intervição } & 25,2 & 3,48 \\ \text { Pós-intervição } & 25,5 & 2,91\end{array}$

Fonte: a pesquisa MEEM: Mini Exame do Estado Mental. $\mathrm{N}=10$

Tabela 5- Pontuação obtida no Questionário SF-36 pré e pós intervenção

\begin{tabular}{lccccc} 
& \multicolumn{2}{c}{ PRÉ } & \multicolumn{2}{c}{ PÓS } & P \\
Capacidade Funcionel & M & DP & M & DP & \\
Aspectos Físicos & 97,56 & 2,51 & 98,67 & 3,32 & 0,347 \\
Dor & 94,44 & 16,67 & 66,67 & 25,00 & 0,051 \\
Estado de Saúde Geral & 78,33 & 20,55 & 62,22 & 3,67 & $0,037^{*}$ \\
Vitaliade & 81,78 & 15,01 & 78,22 & 4,32 & 0,519 \\
Aspectos Sociais & 79,44 & 1,67 & 77,78 & 8,70 & 0,594 \\
Aspectos Emocinais & 77,78 & 15,02 & 98,61 & 4,17 & $0,008^{*}$ \\
Saúde Mental & 100,00 & 0,00 & 100,00 & 0,00 & 1,000 \\
Fonte: pesquisadora. M: média; DP: desvio padrão. P<0,05. N=10 & 59,11 & 14,39 & 69,33 & 2,83 & 0,081
\end{tabular}




\section{artigo}

Julik, A.D.; Miri, A.L.; Bini, A.C.D.; Suckow, P.P.T.; Fonseca, E.G.J.; Moreira, F.F.; Carraro, E.;

Influência da equoterapia na qualidade de vida de idosos caracterizados com a classificação internacional de funcionalidade - CIF

da, com dificuldade leve ou sem dificuldade, inclusive nos fatores ambientais.

Na presente pesquisa também buscou-se descrever o perfil dos idosos, ao ser aplicado uma avaliação contendo dados pessoais, estado cognitivo (MEEM) e qualidade de vida (SF-36). Corroborando com os estudos de Campos et al. (2014) e Araújo e Faro (2014), as amostras possuem capacidade cognitiva preservada e são predominantemente do sexo feminino, isso pode ser explicado pela maior longevidade das mulheres, sua maior adesão a programas de atividade física, bem como maior busca pelos serviços de saúde e adaptação a um estilo de vida saudável.

Nos estudos de Campos et al. (2014) e Araújo e Faro (2014) a renda mensal da amostra é de aproximadamente um salário mínimo, enquanto no presente estudo o perfil são de idosos com uma renda mensal superior (4 mil reais).

Considera-se no presente estudo um público privilegiado, pois são idosos saudáveis, sem alteração cognitiva e praticantes de atividade física regular. Ademais, concluiu-se pela caracterização da CIF qualificadores positivos quanto a função, estrutura, atividade e participação (Domínios I, II e III).

Adversamente a investigação de Araújo e Faro (2014), os idosos da presente pesquisa relataram problemas relacionados a fatores ambientais (CIF-Domínio IV), os quais não se apresentaram homogêneos $\mathrm{e}$ tiveram predomínio nos qualificadores $3 \mathrm{e}$ 4 (grave problema apresentado a completo problema apresentado).

Esses resultados demonstram que satisfação a fatores ambientais e percepção de boa qualidade de vida são subjetivos de indivíduo para indivíduo, e que os domínios da CIF não estão diretamente inerentes um ao outro, pois a presente amostra não demonstrou ter problemas quanto a função, estrutura, atividade e participação, além disso apresenta renda mensal estável, e mesmo assim houve relato de problemas ambientais (medicamentos; produtos e tecnologias gerais para atividades; culturais, recreativas e esportivas e serviços de saúde.)

Kim (2018) em um estudo clínico, com 51 indivíduos, divididos em quatro grupos experimentais adultos mais jovens com cavalo terapêutico, adultos mais velhos com cavalo terapêutico, adultos mais jovens com cavalo simulados, e idosos com cavalo simulados. Foi avaliado com a eletromiografia músculos de estabilidade postural, em que consistia a sessão de: caminhada $80 \mathrm{~m} /$ minuto, trote lento $135 \mathrm{~m} /$ minuto, rápido trote $159 \mathrm{~m} /$ minuto. Afirmaram que os exercícios da equoterapia foram mais vantajosos para os adultos mais velhos e idoso, do que em relação aos adultos jovens. Reduzindo as alterações no equilíbrio, consequentemente, reduzindo os riscos de quedas. Observa-se em nosso estudo que função vestibular, função proprioceptiva, resistência física geral capacidade aeróbica, mobilidade generalizada das articulaçôes, força, tônus e resistência de todos os músculos do corpo estão normais nos idosos, diminuindo assim os riscos de quedas.

Kim e Lee (2015) investigaram o efeito da simulação de equitação no equilíbrio e na ativação do tronco, buscando fornecer evidências dos benefícios terapêuticos do exercício. Recrutaram 30 idosos e os dividiram aleatoriamente em grupo controle e grupo experimental, sendo que apenas o grupo experimental realizou o exercício. $\mathrm{O}$ protocolo de exercícios teve duração de 20 minutos, 5 vezes por semana, por 8 semanas e foi associado a terapia convencional. Ao analisarem medidas de ativação muscular e limites de estabilidade, os autores sugerem que o exercício de equitação aumenta a ativação muscular e a estabilidade dinâmica em relação ao controle, ainda afirmam que esta pode ser uma abordagem eficaz na redução do risco de quedas.

Cho (2017) identificou em 31 idosos saudáveis, divididos aleatoriamente, os efeitos da equitação real e do exercício mecânico de equitação sobre a estabilidade psicológica, através da potência alfa relativa (eletroencefalograma). O programa de exercícios foi conduzido por 25 minutos, 2 vezes semanais, durante 12 semanas. Consistiu em exercícios de aquecimento, equitação (treinamento em vários planos e direções) e desaquecimento (alongamento, treino respiratório). Os resultados demonstraram que o grupo equitação real obteve aumento na potência alfa relativamente rápido, enquanto o grupo mecânico um aumento da potência alfa relativamente lento, sugerindo assim, que ambos os grupos possuem um efeito positivo na estabilidade psicológica em idosos.

Programas de equoterapia são uma excelente ferramenta terapêutica, melhorando o equilíbrio estático e dinâmico, função motora grossa e fina, marcha, espasticidade, coordenação motora, reduz do risco de quedas e gera efeitos positivo sobre a estabilidade psicológica, apresentando benéficios da prática de equoterapia em idosos. A presente investigação corrobora com esses estudos quanto a efetividade da equoterapia em idosos ${ }^{8,17,18,19}$.

Na presente análise, foi encontrado diferença estatística no SF-36, pré e pós intervenção na dor $(p=0,037)$ e nos aspectos sociais $(p=0,008)$. Logo, ao atuar nesses aspectos, a equoterapia demostra-se uma alternativa terapêutica capaz de manter/ melhorar a qualidade de vida. Oque corrobora em um relato de caso de Santos (2019), um paciente com 85 anos de idade, praticou equoterapia por sete semanas. Cada sessão da terapia consistiu em 30 minutos de atividades para promoção de equilíbrio, postura e autoconfiança. Antes da primeira, e ao final da última sessão de equoterapia o idoso respondeu ao IDATE (Inventário de Ansiedade Traço- Estado) e ao WHOQOL- Bref (World Health Organization Quality of Life- Bref). Concluiu-se que a prática de equoterapia melhorou os escores da qualidade de vida desse idoso avaliado.

Acredita-se que não houve diferença estatística em outras variáveis do SF-36 (capacidade funcional, aspectos físicos, estado de saúde geral, vitalidade, aspectos emocionais e saúde mental) por se tratar de uma população-alvo sem alguma deficiência e/ ou problema relevante instalado.

No estudo de Mello (2018) participaram para análise do tronco 20 participantes, 15 do gênero feminino e cinco do masculino, com média de 69 anos. E para análise da mastigação 17 participantes, 13 do gênero feminino e 4 do masculino, com média de 66 anos. Foram realizados 11 atendimentos de Equoterapia, uma vez 
por semana, com duração de 30 minutos. Utilizando o eletromiografo para análise, foi concluido que Equoterapia promove alterações na atividade da musculatura de idosos, sendo os músculos do tronco ativados, de maneira crescente e até mesmo do recrutamento dos músculos da mastigação, o que aumenta significativamente a qualidade de vida dos idosos.

A implementação de um grupo controle, número amostral maior e análises mais amplas são potenciais ideias para novos estudos e/ou prosseguimento deste.

\section{CONCLUSÃO}

O perfil de idosos do presente estudo são predominantemente do sexo feminino, casadas, idade média de 70,6 anos, com sobrepeso. $\mathrm{Na}$ caracterização amostral pela CIF constatou-se nos Domínios I, II e III qualificadores predominantemente em 0 e 2 , ou seja, ne- nhum problema apresentado a moderado problema apresentado. Já no Domínio IV, os valores foram predominantes entre 3 e 4 , variando desde grave problema apresentado a completo problema apresentado. No SF-36 houve diferença estatística entre pré e pós intervenção na dor e nos aspectos sociais.

Conclui-se que a terapia assistida com equinos em idosos é uma alternativa terapêutica efetiva na manutenção e/ou melhora da qualidade de vida.

\section{REFERÊNCIAS}

1. Rey-Mermet A. \& Gade, M. Inhibition in aging: What is preserved? What declines? A meta-analysis. Psychon Bull Rev. 2018; 25:1695-1716.

2. Duque A, Peixoto MV, Lima S, Goes MA, Santos A, Araújo KC. et al. Analysis of the relationship between life expectancy and social determinants in a north-eastern region of Brazil, 20102017. Geospatial Health. 2018; 13(2).

3. Campos FOS. A management of public health in the municipality of Salvador, in the access of the elderly population, at the level of primary care, in front of a neoliberal government. Universidade Católica do Salvador. 2020.

4. Suzman R, Beard JR, Boerma T, Chatterjj. S. Health in an ageing world- What do we know. Lancet; 2015; 385: 484-6.

5. Czibere I, Rácz A, Szilvási H, Szikszai Z, Imre S. Examination of life quality, mental conditions and cognitive status of people over the age of 90: Results of a Hungarian local research.Cent Eur J Public Health. 2019; 27(1):17-23.

6. Certo A, Sanhez K, Galvão AM, Fernades H. A síndrome da fragilidade nos idosos: revisão da literatura. In Actas de Gerontologia: Congresso Português de Avaliação e Intervenção em Gerontologia Social. 2016; 2(1).1-11

7. Pohl P, Carlsson G, Bunketorp Käll L, Nilsson M, Blomstrand C. A qualitative exploration of post-acute stroke participants' experiences of a multimodal intervention incorporating horseback riding. PloS one. 2018; 13(9).

8. Kim MJ, Kim T, Oh S, Yoon B. Equine Exercise in Younger and Older Adults: Simulated Versus Real Horseback Riding. Perceptual and Motor Skills. 2018;125(1):93-108.

9. Livingston-Thomas J, Nelson P, Karthikeyan S, Antonescu S, Jeffers MS, Marzolini S. et al. Exercise and Environmental Enrichment as Enablers of Task-Specific Neuroplasticity and Stroke Recovery. Neurotherapeutics. 2016;13(2) 395-402.

10. Ruaro, J.F. Proposição e aplicação de um core set da Classificação Internacional de Funcionalidade, Incapacidade e Saúde (CIF) para saúde física de idosos. Tese - Universidade Federal do Rio Grande do Norte, Natal, 2014.

11. Folstein MF, Folstein SE, Mchugh PR. "Mini-mental state". A practical method for grading the cognitive state of patients for the clinician. J Psychiatr Res. 1975; 12(3):189-98.

12. Lins L, Carvalho FM. Pontuação total do SF-36 como uma medida única de qualidade de vida relacionada à saúde: revisão do escopo. SAGE Open Medicine . 2016.

13. Ciconelli RM, Ferraz MB, Santos W, Meinão I, Quaresma MR. Tradução para a língua portuguesa e validação do questionário genérico de avaliação de qualidade de vida SF-36 (Brasil SF-36). Rev Bras Reumatol. 1999; 39 (3). 143-150.

14. ANDE-BRASIL [Associação Nacional de Equoterapia]. In: Curso Básico de Equoterapia, São Paulo, 2004.

15. Campos ACV, Cordeiro EC, Rezende GP, Vargas AMD. Quality of life of elderly practitioners of physical activity in the context of the family health strategy. Texto contexto - enferm. 2014; 23(4). 889-897.

16. Araújo CLO, Faro ACM. Condições de saúde e funcionalidade dos idosos do Vale Paraíba, São Paulo, Brasil. Enfermería Global. 2014; 33. 100-115.

17. Kim SG, Lee JH. The effects of horse riding simulation exercise on muscle activation and limits of stability in the elderly. Archives of Gerontoly and Geriatrics. 2015; 60. 62-65.

18. Cho SH. Effects of horseback riding exercise on the relative alpha power spectrum in the elderly. Archives of Gerontoly and Geriatrics. 2017; 70.141-147.

19. Stergiou A, Tzoufi M, Ntzani E, Varvarousis D, Beris A, Ploumis A. Therapeutic Effects of Horseback Riding Interventions: A Systematic Review and Meta-analysis. Am J Phys Med Rehabil. 2017; 96(10):717-725.

20. Santos ACG, Araújo AMS, Orlando DR, Lobo-Júnior AR, Andrade E.F. Efeitos da equoterapia sobre a ansiedade e qualidade de vida de um idoso institucionalizado: um relato de caso. ENCICLOPÉDIA BIOSFERA, Centro Científico Conhecer - Goiânia. 2019;16(30). 231.

21. Mello EC. Efeitos da equoterapia sobre as atividades eletromiográficas dos músculos do tronco e da mastigação em praticantes idosos. 2018. 86f. Dissertação (Mestrado em Ciências da Saúde) - Programa de Pós-Graduação em Ciências da Saúde, Universidade Federal do Triângulo Mineiro, Uberaba, 2018. 